\title{
Sex Differences in Time Perception during Self-paced Running
}

\author{
NICHOLAS J. HANSON ${ }^{\dagger 1,2}$, and JANET BUCKWORTH $\$ 1,3$ \\ 1 The Ohio State University, Columbia, OH, USA; ${ }^{2}$ Department of Human \\ Performance and Health Education, Western Michigan University, Kalamazoo, \\ MI USA; ${ }^{3}$ Department of Kinesiology, University of Georgia, Athens, GA, USA
}

†Denotes graduate student author, $\ddagger$ Denotes professional author

\begin{abstract}
International Journal of Exercise Science 9(4): 514-523, 2016. Time perception during exercise may be affected by chosen intensity, and may also affect enjoyment of exercise and subsequent long-term adherence. However, little is known about how individuals perceive the passage of time during exercise, or if factors such as sex are influential. The purpose of this study was to determine if there are sex related differences in perception of time during a bout of exercise in experienced runners. Twenty-two recreational runners (11 men, 11 women) participated in a bout of treadmill running where they were allowed to select their intensity. Sixty second prospective time estimations were taken before, during (at 33\%, 66\% and $90 \%$ of the completed distance), and after the run. Heart rate (HR) was also recorded throughout. The women $(M=91.9, S D=3.3)$ ran at a significantly higher percentage of their maximum HR than the men $(M=86.5, S D=6.4 ; p=0.022)$, choosing to run at a higher relative intensity than the men when given the opportunity to self-pace. The women had relatively lower time estimations overall, showing that they perceived time to be passing by more slowly compared to the men. These results may help to explain sex related differences in exercise adherence.
\end{abstract}

KEY WORDS: self-pacing, prospective, time perception, teleoanticipation, running

\section{INTRODUCTION}

The perception of time is a part of the human experience and is essential in everyday behavior (41). The temporal experience of humans is also part of an individual's specific relationship to his or her own environment. Under differing circumstances and between individuals, time can be perceived as faster or slower than objective measures. This experience of time is an integration of parallel chains of events, both external and internal, and depends on a highly functioning nervous system capable of this integration (11).
Simple decisions that we all make on a daily basis, such as waiting for elevators or taking stairs are based on an individual's perception of time passage (41).

The two commonly described paradigms of time perception research are retrospective and prospective. The retrospective paradigm involves having an individual estimate the amount of time that they believe has passed. In the prospective paradigm, participants know that they will be asked to judge the duration of a time period. Research has shown that estimation of time retrospectively and prospectively 
uses different neural processes; while retrospective time estimation uses primarily stored memory processes, prospective estimation utilizes attentional control and allocation (42). The method commonly used to investigate prospective time estimation differences $(6,23)$ is to divide the estimated time (subjective time determined by the subject) by the objective time. For example, if someone reports that 60 seconds have passed after only 50 seconds, this ratio of $0.83(50 / 60)$ represents the perception that subjective time is passing slower than objective time.

Eson and Kafka (11) state that disturbances in the experience of time generally come from (a) distorted external events, (b) physiological disturbances, such as varying heart and respiration rates, and (c) a defective or malfunctioning mechanism that is involved with the integration of the above two factors. Studies have shown that a host of personal factors can affect the perception of time. Cognitive functions such as attention, memory (both long and short-term), drive states, mood, emotion, anxiety and personality have all been shown to affect time perception in some way $(8,30,41)$. Other studies have shown that age, sex, and metabolism are also factors that can in some way influence how people perceive the passage of time $(6,19)$. The effect of sex on time perception has shown conflicting results over the years (5, $12,26)$. However, a recent study published on the topic showed that women tend to underestimate prospective time estimations compared to men, suggesting they may perceive time to be passing by more slowly (17).

Exercise creates a physiological disturbance to the human body, and can alter heart and respiration rates, mood, emotions and metabolism. Little is known, however, about the effects of exercise on the perception of time. Every bout of exercise must have a start and a finish, and the knowledge of that endpoint can influence pacing, attentional focus and motivation. This concept is known as teleoanticipation (38) and involves both feedback and feedforward regulation of metabolic reserves in an attempt to reach the endpoint without failure. St Clair Gibson et al. (31) suggested that humans have an "internal clock" with scalar time scales used by the brain to cover a certain distance without catastrophic failure. The person is thereby able to adjust power output and the rate of metabolic processes as needed throughout the exercise or race. Pacing strategies of athletes depends on physiological capacity, duration or distance of the event, exercise mode, level of competition, environment, motivation and experience of the athlete (14). Very few studies could be located investigating the perception of time during exercise or involving athletes and none of these have investigated sex differences or chosen intensity $(24,34,37,39)$. It is very likely that the intensity level chosen by individuals affects how they perceive the passage of time, and that knowledge of an endpoint impacts the chosen intensity as well.

The perception of time during exercise, which may be affected by intensity level, is also likely to influence the overall level of enjoyment of exercise, and could have implications for long-term adherence. However, little is known about how individuals perceive the passage of time during exercise, or if any differences exist between men and women. Therefore, the purpose of this study was to explore the 
perception of time during self-paced exercise in experienced recreational runners, and to determine if there are any sex-related differences present. We hypothesized that the women in the study would underestimate time durations as compared to men, and these changes would persist throughout the exercise bout. We also hypothesized that there would be no differences in the self-selected intensity between the men and women.

\section{METHODS}

\section{Participants}

Volunteers for this study were 22 recreational runners (11 men). Written informed consent was obtained for all participants, and the study was approved by the Institutional Review Board. The descriptive characteristics of the participants, separated by sex, are shown in Table 1. All interested individuals met the inclusion criteria, which required them to be "low risk" according to the risk stratification guidelines suggested by the American College of Sports Medicine (35), run a minimum average of $16 \mathrm{~km}$ per week for six months leading up to the testing, and be healthy and injury-free at the time of testing. Participants were recruited from the local student body, the university running club, and from visitors and customers of the local running store.

\section{Protocol}

Laboratory Visit \#1. After signing the consent form, the participants were asked to complete a $\mathrm{VO}_{2}$ max test in order to assess their level of cardiorespiratory fitness.
Table 1. Comparison between men and women participants, listed as M (SD).

\begin{tabular}{|c|c|c|c|}
\hline Variable & Men & Women & $p$ \\
\hline Age (years) & $28.6(6.4)$ & $24.5(5.5)$ & .116 \\
\hline BMI $\left(\mathrm{kg} \cdot \mathrm{m}^{-2}\right)$ & $23.3(2.9)$ & $21.2(2.2)$ & .071 \\
\hline $\begin{array}{l}\text { Years of running } \\
\text { experience }\end{array}$ & $10.1(5.7)$ & $8.7(4.7)$ & .553 \\
\hline $\begin{array}{l}\text { Distance run during } \\
\text { testing }(\mathrm{km})\end{array}$ & 7.7 (1.9) & $5.6(1.0)$ & .003 \\
\hline Time to complete run & $34: 27(9: 38)$ & 27:55 (3:44) & .050 \\
\hline $\mathrm{VO}_{2} \max \left(\mathrm{ml} \cdot \mathrm{kg}^{-1} \cdot \mathrm{min}^{-1}\right)$ & $57.9(7.8)$ & $48.9(3.5)$ & .006 \\
\hline
\end{tabular}

True One 2400 metabolic cart (ParvoMedics, Sandy, UT) was used for analyzing expired gases, and a Polar chest strap was used to monitor heart rate (HR; Polar Electro Oy, Finland). The metabolic system was calibrated before each use with a 3L syringe. After a five minute warm-up at a self-selected pace, a Modified AstrandSaltin $\mathrm{VO}_{2}$ max protocol was implemented (21). For this protocol, a speed slightly higher than each participant's normal training speed was chosen, and was clamped for the entirety of the test. The grade, initially at $0 \%$, was increased by $2 \%$ every two minutes until volitional exhaustion. The two highest consecutive values for relative $\mathrm{O}_{2}$ consumption were averaged for determination of $\mathrm{VO}_{2} \max$. Tests were considered to be maximal if two of the following three criteria were obtained: (1) RPE: $\geq 18$; (2) HR: within 10 bpm of the participant's age predicted maximal HR (where $\mathrm{HR}_{\max }=220$ - age); (3) $\mathrm{VO}_{2}$ plateau: difference between peak relative $\mathrm{VO}_{2}$ value and the value in the preceding 15 seconds of $2.0 \mathrm{ml} \cdot \mathrm{kg}^{-1} \cdot \mathrm{min}^{-1}$ or less.

Laboratory Visit \#2. This visit was scheduled a minimum of 48 hours after visit \#1 but no more than one week later. Participants were fitted with a Polar HR monitor, the purpose of which was to use 
as an indicator of their self-selected intensity by comparing their $\mathrm{HR}$ during exercise as a percentage of their maximal HR. They were asked to warm up on the treadmill for five minutes at a self-selected pace. After the warm up period, their first time estimation was performed, and they were given approximately 5 minutes to prepare for the next part of the lab visit. The participants were then asked to run for a predetermined distance. This was calculated based on the running history of each subject, obtained from the first visit, and was specifically based on $75 \%$ of their daily run. For example, if the participant noted in their running history that their typical weekday run was $10 \mathrm{~km}$, the distance they would be asked to run would be $75 \%$ of that distance, or $7.5 \mathrm{~km}$. They were told to run at a pace of their choosing, and they would be allowed to adjust the pace at any point in the trial. However, they were not allowed to see the treadmill display at any time during the testing. They were given no feedback as to the distance left to complete, but were simply told that they were done when they reached the endpoint.

Prospective Time Estimation. Participants were asked to estimate time duration by verbally expressing a "start" and "end" when they believed that a 60 second period of time had elapsed. This time production method used to assess perception of time was similar to that used by EspinosaFernandez and colleagues (12); however, in the current study the researcher was in control of the stopwatch rather than the subject. Using a stopwatch, the actual time passed was recorded and compared to the estimated time. Participants were not told what their estimated time was during the experiment, as feedback could have affected performance on this task (15). They performed five 60-second time estimations during Laboratory Visit \#2: before testing, three times during the run (at 33\%, 66\% and $90 \%$ of the distance covered) and once after testing. There were no clocks in the room, and the participants were not allowed to wear a wristwatch at any point during data collection.

The following verbiage was used to describe the time estimations to participants: I would like you to estimate a 60 second period of time. To begin, you must verbally express that you are ready by saying the word "start" aloud, and I will begin the time collection. When you think that 60 seconds has passed, you must say "stop." This is the point in which I will end time collection.

Laboratory environment. Consistency throughout testing was a priority with this study. For all laboratory visits, the same environment was created for the participants to the best of our ability. The same treadmill was used each session, and the primary investigator was present for all testing sessions. The laboratory door was kept closed to ensure that nobody entered the room during testing.

\section{Statistical Analysis}

Statistical analyses were performed using IBM SPSS Statistics for Windows (Version 22.0. Armonk, NY: IBM Corp). A mixed ANOVA (analysis of variance) with sex as a between-subjects factor and time as a within-subjects factor was used to determine the amount of variance in time estimation ratios between men and women, as well as the point in which the time estimations were taken (before, during and after testing). Independent $t$-tests were performed in order to determine if any 
simple effects were present. The significance level for all analyses was set $a$ priori at $p<.05$. Partial eta squared was provided as an effect size for the mixed ANOVA, and Cohen's $d$ was provided as an effect size for independent t-tests.

\section{RESULTS}

Comparisons between men and women participants are reported in Table 1 . Independent $\mathrm{t}$-tests showed that distance run during testing and $\mathrm{VO}_{2}$ max were significantly different between the men and women $($ all $p<.005)$.

Time estimation ratios for men and women participants before, during and after the testing session are displayed in Figure 1. There was a main effect of sex; the women had significantly lower time estimation ratios compared to the men $(F(1,20)=6.592$, $\left.p=.018, \eta_{\mathrm{p}}{ }^{2}=.248\right)$. There was not a significant main effect of time and no interaction effects were present $\left[F(2.190,43.809)=2.155, p=.125, \eta_{p}^{2}=.097\right.$, and $F(2.190,43.809)=1.092, p=.349, \eta_{p}^{2}=$ .052, respectively, with Greenhouse-Geisser corrections], showing that there were no differences in time estimation ratios between time points (before, during or after) and that no condition was significantly different from all others.

Heart rate was used as an indicator of participants' self-selected intensity during the testing session (Figure 2). Independent t-tests showed that women in the study ran at a significantly higher percentage of their maximum $\mathrm{HR}$ compared to men when analyzing both the average $[t(20)=2.488, p$ $=.022, d=1.061]$ and the final $[t(20)=2.149$, $p=.044, d=.917]$ recorded HR. Over the course of the entire run, the mean HR was
$86.5 \%$ for the men and $91.9 \%$ for the women. The final HR assessment was $90.2 \%$ for the men and $94.8 \%$ for the women.

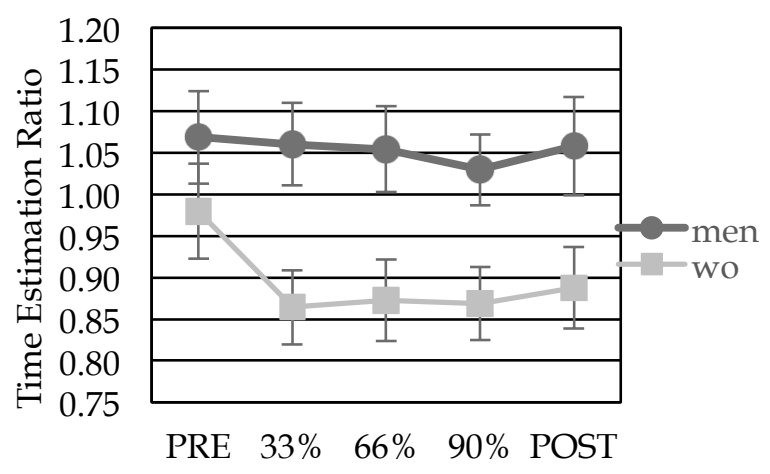

Figure 1. Differences in time estimations between men and women. Values are means \pm SE. A significant main effect of sex was present $(p<.05)$.

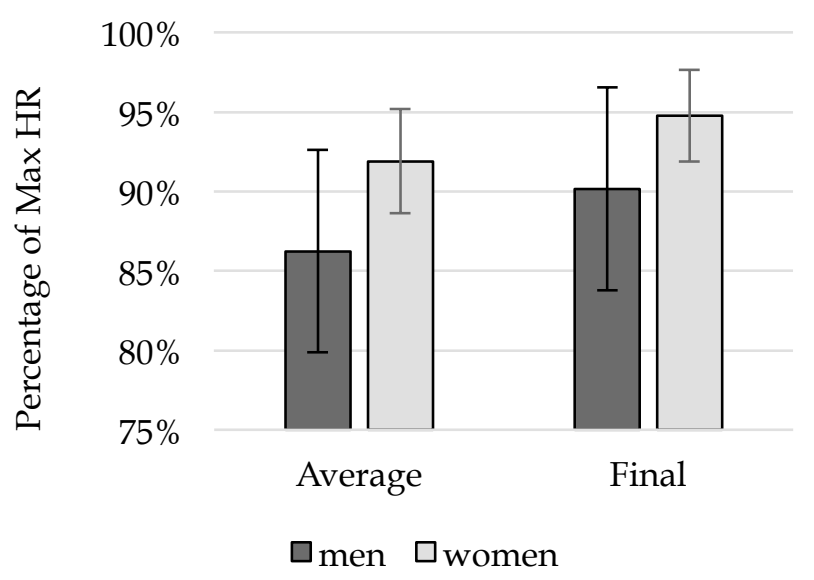

Figure 2. Differences in mean and final percentage of maximum heart rate (HR) between men and women participants. Values are means \pm SD. Men: average percentage of $\max \mathrm{HR}: M=86.5, S D=6.4$, final percentage of $\max$ HR: $M=90.2, S D=6.4$. Women: $M=91.9, S D=3.3$ and $M=94.8, S D=2.9$, respectively.

\section{DISCUSSION}

Although the women in this study had lower levels of cardiovascular fitness compared to the men, both groups fall into the $90^{\text {th }}$ percentile for their average age 
groups (2). And even though the women ran a shorter distance during testing, the time that they ran during the test was not significantly different than the time that the men ran. Lastly, despite the low subject numbers, differences in time perception were elicited. For these reasons we feel that it is appropriate to compare the men and women in this study.

The major finding in this study was the difference in perception of time between men and women participants during a bout of self-paced exercise. Previous studies have suggested differences between men and women but this is the first to show these differences persist through a bout of exercise. A prospective time estimation ratio above 1.0 represents a feeling of time moving by quickly, whereas a ratio less than 1.0 indicates time as progressing relatively slowly. The women had an average time estimation ratio of .895 (SD = .162) and the men had an average of 1.054 $(\mathrm{SD}=.172)$. These ratios equate to average 60 second time estimations of 54 seconds for the women and 63 seconds for the men. This showed that the women in this study, compared to men, experienced time as moving by more slowly; these differences were present before, during and after each run. If these results were applied over the entire exercise bout, women would have experienced the average 30-minute exercise bout as lasting over 3 minutes longer than it did, while men would have experienced the same duration bout as taking about 1.5 minutes less time than it did. It is not known whether knowledge of this information could affect the behavioral choice to exercise, but would be an interesting area for a future study to address.
The results seen in the present study are in line with those of a meta-analytic review of sex differences in time estimation (5). The researchers note that sex differences in duration judgments are moderated mainly by the method used, either the retrospective or prospective paradigm. In the present study, the method used was prospective estimations of time in which we requested participants to produce 60 second time intervals. These were chosen because they are more affected by attention level than retrospective estimation, which are more reliant on memory. Previous research has shown exercise to affect direction and focus of attention $(20,29,33)$. When looking specifically at the prospective time estimation studies in the meta-analysis, Block et al. discovered 74 relevant articles to review, none of which compared time estimates between men and women during exercise. However, there were sex differences in estimations; specifically, women tended to make shorter productions of time compared to men. The results of the meta-analysis, along with those of the present study, support the notion that women focus their attention more on time than men and accumulate "temporal units" at a faster rate.

There may be inherent sex differences in the perception of time, but age may affect these differences. Espinosa-Fernandez et al. (12) also asked men and women to prospectively estimate a 60 second period of time. They did not find a main effect of sex in their study; there were, however, sex differences between age groups. Men 11 through 40 years of age had larger time estimation ratios than women in the same age range. For participants aged 41 through 70 years, the opposite was true; women increased time interval ratios and 
men decreased. In the current study, the age range for the men was 19 to 40 and for the women was 19 to 35 . This age range is very similar to the range reported by Espinosa-Fernandez et al. (12) that resulted in larger time estimation ratios by the men compared to the women. We have shown that in our sample, this sex difference in time perception is observed at rest and during exercise.

Another finding from the current study was the difference in self-selected intensity between men and women participants. It was discovered that the women in the study had significantly higher average and final HR compared to the men. As most other studies investigating teleoanticipation have either only involved men $(1,9,13,40)$ or did not report sex differences in HR data $(3,4,28)$, these results can only be compared to a limited number of relatable studies. Faulkner et al. (14) used men and women as participants and they did not discover any sex differences or interactions in HR response in either running condition in their study (7 mile run or half-marathon). Dasilva and colleagues (10) asked men and women to walk at a self-selected pace on a laboratory treadmill. They found no significant sex differences in walking speed, RPE, HR or percentage of HR, even though the men were consuming significantly more oxygen than the women $(p<.05)$. March et al. (27) showed that women runners held a more consistent pace throughout a marathon compared to men. Perhaps the women in our study were able to maintain a faster pace throughout testing without feeling the need to decrease speed. Future research should be performed to explore these differences. Regardless, both the men and the women in this study exercised at a fairly high intensity level. Future studies may focus on controlling for intensity level, as this may affect the perception of time for both sexes.

Previous research has shown that body temperature and metabolism can also affect the perception of time, and a fairly consistent relationship has been determined $(16,22,32,36)$. Hancock et al. (19) showed that when brain temperature was increased artificially with a heated helmet, 41-second prospective time estimations were significantly lower. The increased brain temperature essentially acted to "slow down" the perceived time in those individuals. When exercising at a relatively high intensity, core temperature in both men and women is known to increase. Regardless of environmental temperature, when people exercise, cutaneous vasoconstriction occurs initially. This acute reduction in skin blood flow is elicited by increased vasoconstrictor system activity; as exercise continues, the vasodilator system is activated in an attempt to keep core temperatures low by redirecting blood flow to the cooler periphery (18). During exercise, it is the goal of the body to maintain a proper core temperature, but fatigue and dehydration can lead to a decreased stroke volume and an increase in core temperature. Increases in body temperature have been shown to affect the perception of time, which may explain the slight drop in time estimations during exercise in the current study. Since our participants were running at a relatively high percentage of their maximum HR, this effect on perception of time could be partially due to increased core temperature as the bout of exercise progressed. This is merely speculative as this variable was not measured as part of this study, but is 
something that future studies in this area should consider assessing.

Previous research has shown that adolescent males and females have similar levels of physical activity until roughly the age of seventeen years (7). At this age, women seem to have diminishing levels of activity compared to men. Adolescent men also tend to have higher levels of strengthtraining activity and high-intensity exercise compared to women (25). Even into adulthood, men are more likely to implement strength-training activity and regular sustained activity into their daily exercise routines. Additionally, women in the United States are about 14 percent more likely than men to be sedentary (25). The differences in self-selected intensity and time perception between the men and women in the current study may help to explain these disparities. A common barrier to exercise is perceived lack of time, and women's perception of time passing slowly during exercise could contribute to less willingness to spend time in this activity. In addition, women may have an inherently different perception of time compared to men. This study showed that this difference was present not only during exercise but also at rest. Whether or not this difference is due to the specific population tested in the current study (recreational runners) or if can be altered is yet to be determined. Furthermore, the difference in time perception between aerobic and anaerobic activity has yet to be explored, as well as the difference between regular exercisers and non-exercisers.

There were some limitations to this study. The participants in this study were recreational runners who took part in a single bout of treadmill exercise. The modality used, along with intensity and duration, may all affect the perception of time during exercise. The treadmill was chosen as a modality because the participants were runners. Use of a cycle ergometer, elliptical or other typical lab equipment could possibly affect the results. The participants were allowed to choose their own intensity because runners are nearly always allowed to choose their own pace in training or a race; if we prescribed their speed or intensity the results may be different. Also, these active individuals may have chosen a different self-selected exercise intensity than a non-athletic population. The duration of each run was influenced by the participants' chosen speed, but the distance that was calculated for them to run was based off of their training. Regardless, intensity level is likely to influence the perception of time. It is probable that a host of behavioral, psychological, physiological, and environmental factors play a part in the perception of time, and the present study was unable to address all of these factors in an attempt to adopt a parsimonious methodology. These results add to the limited body of research investigating exercise and the perception of time, but future studies should expand on these findings and attempt to determine the causes and moderators. Possible areas to explore would be the time of day, menstrual cycle, controlling for intensity, as well as the measurement of core body temperature, anxiety and personality.

The following conclusions are warranted from the results of this study: when given the opportunity to self-pace, these experienced female runners chose to run at a higher relative intensity than the men. The women also had relatively lower time estimations, showing that they perceived 
time to be passing by more slowly compared to the men before, during and after a bout of exercise. The women's perception of time passing by more slowly during exercise may have implications for exercise adoption and adherence, and should be examined in novice compared to experienced female runners.

\section{REFERENCES}

1. Albertus Y, Tucker R, St Clair Gibson A, Lambert EV, Hampson DB, Noakes TD. Effect of distance feedback on pacing strategy and perceived exertion during cycling. Med Sci Sports Exerc 37(3): 461-468, 2005.

2. American College of Sports Medicine. ACSM's health-related physical fitness assessment manual. Lippincott Williams \& Wilkins; 2013.

3. Baden DA, McLean TL, Tucker R, Noakes TD, Gibson ASC. Effect of anticipation during unknown or unexpected exercise duration on rating of perceived exertion, affect, and physiological function. Br J Sports Med 39(10): 742-746, 2005.

4. Baden DA, Warwick-Evans L, Lakomy J. Am I Nearly There? The effect of anticipated running distance on perceived exertion and attentional focus. J Sport Exercise Psy 26(2): 215-231, 2004.

5. Block RA, Hancock PA, Zakay D. Sex differences in duration judgments: A meta-analytic review. Mem Cognit 28(8): 1333-1346, 2000.

6. Block RA, Zakay D, Hancock PA. Human aging and duration judgments: A meta-analytic review. Psychol Aging 13(4): 584-596, 1998.

7. Caspersen CJ, Pereira MA, Curran KM. Changes in physical activity patterns in the United States, by sex and cross-sectional age. Med Sci Sports Exerc 32(9): 1601-1609, 2000.

8. Claridge G. The excitation-inhibition balance in neurotics. Experiments in Personality 2: 107-154, 1960.
9. Coquart JB, Garcin M. Knowledge of the endpoint: effect on perceptual values. Int J Sports Med 29(12): 976-979, 2008.

10. Dasilva SG, Guidetti L, Buzzachera CF, Elsangedy HM, Krinski K, De Campos W, Goss FL, Baldari C. Gender-based differences in substrate use during exercise at a self-selected pace. J Strength Cond Res 25(9): 2544-2551, 2011.

11. Eson ME, Kafka JS. Diagnostic implications of a study in time perception. J Gen Psychol 46(2): 169183, 1952.

12. Espinosa-Fernández L, Miró E, Cano MC, Buela-Casal G. Age-related changes and gender differences in time estimation. Acta Psychol (Amst) 112(3): 221-232, 2003.

13. Eston R, Stansfield R, Westoby P, Parfitt G. Effect of deception and expected exercise duration on psychological and physiological variables during treadmill running and cycling. Psychophysiology 49: 462-469, 2012.

14. Faulkner J, Parfitt G, Eston R. The rating of perceived exertion during competitive running scales with time. Psychophysiology 45(6): 977-985, 2008.

15. Fraisse P. Learning of the estimation of duration and its bearings. L'année psychologique 71(2): 371-379, 1971.

16. Gilliland AR, Hofeld J, Eckstrand G. Studies in time perception. Psychol Bull 43(2): 162-176, 1946.

17. Glicksohn J, Hadad Y. Sex differences in time production revisited. J Individ Dif 33(1): 35-42, 2012.

18. Gonzalez-Alonso J, Crandall CG, Johnson JM. The cardiovascular challenge of exercising in the heat. J Physiol 586(1): 45-53, 2008.

19. Hancock PA. Body temperature influence on time perception. J Gen Psychol 120(3): 197-216, 1993.

20. Hanson NJ, Buckworth J. The effect of endpoint knowledge on perceived exertion, affect, and attentional focus during self-paced running. J Strength Cond Res 29(4): 934-941, 2015. 
21. Hawkins MN, Raven PB, Snell PG, StrayGundersen J, Levine BD. Maximal oxygen uptake as a parametric measure of cardiorespiratory capacity. Med Sci Sports Exerc 39(1): 103-107, 2007.

22. Hoagland $\mathrm{H}$. The physiological control of judgments of duration: evidence for a chemical dock. J Gen Psychol 9: 267-287, 1933.

23. Hornstein A, Rotter G. Research methodology in temporal perception. J Exp Psychol 79(3p1): 561564, 1969.

24. Lambourne K. The effects of acute exercise on temporal generalization. Q J Exp Psychol 65(3): 526540, 2012.

25. Lox CL, Ginis KAM, Petruzzello SJ. The Psychology of Exercise. 2nd ed. Scottsdale, Arizona: Holcomb Hathaway; 2006.

26. MacDougall R. Sex differences in the sense of time. Science 19(487): 707-708, 1904.

27. March DS, Vanderburgh PM, Titlebaum PJ, Hoops ML. Age, sex, and finish time as determinants of pacing in the marathon. J Strength Cond Res 25(2): 386-391, 2011.

28. Mauger AR, Sculthorpe N. A new VOmax protocol allowing self-pacing in maximal incremental exercise. Br J Sports Med 46(1): 59-63, 2012.

29. Okwumabua T. Psychological and physical contributions to marathon performance: An exploratory investigation. J Sport Behav 8(3): 163171, 1985.

30. Siegman AW. Anxiety, impulse control, intelligence, and the estimation of time. J Clin Psychol 18(1): 103-105, 1962.

31. St Clair Gibson A, Lambert EV, Rauch LH, Tucker R, Baden DA, Foster C, Noakes TD. The role of information processing between the brain and peripheral physiological systems in pacing and perception of effort. Sports Med 36(8): 705-722, 2006.

32. Stern MH. Thyroid function and activity, speed, and timing aspects of behaviour. Can J Psychol 13(1): 43-48, 1959.
33. Summers JJ, Sargent GI, Levey AJ, Murray KD. Middle-aged, non-elite marathon runners: A profile. Percept Mot Skills 54(3): 963-969, 1982.

34. Tamm M, Jakobson A, Havik M, Burk A, Timpmann S, Allik J, Ööpik V, Kreegipuu K. The compression of perceived time in a hot environment depends on physiological and psychological factors. Q J Exp Psychol 67(1): 197-208, 2014.

35. Thompson WR, Gordon NF, Pescatello LS. ACSM's Guidelines for Exercise Testing and Prescription. 8th ed. Philadelphia, PA: Lippincott Williams \& Wilkins; 480, 2009.

36. Thor DH. Diurnal variability in time estimation. Percept Mot Skills 15(2): 451-454, 1962.

37. Tobin S, Grondin S. Time perception is enhanced by task duration knowledge: Evidence from experienced swimmers. Mem Cognit: 1-13, 2012.

38. Ulmer HV. Concept of an extracellular regulation of muscular metabolic rate during heavy exercise in humans by psychophysiological feedback. Experientia 52(5): 416-420, 1996.

39. Vercruyssen M, Hancock P, Mihaly T. Time estimation performance before, during, and following physical activity. J Hum Ergol (Tokyo) 18(2): 169-180, 1989.

40. Wittekind AL, Micklewright D, Beneke R. Teleoanticipation in all-out short-duration cycling. Br J Sports Med 45(2): 114-119, 2011.

41. Wittmann M. The inner experience of time. Philos T Roy Soc B 364(1525): 1955-1967, 2009.

42. Zakay D. Relative and absolute duration judgments under prospective and retrospective paradigms. Atten Percept Psychophys 54(5): 656-664, 1993. 